Article

\title{
Precipitant-Free Crystallization of Protein Molecules Induced by Incision on Substrate
}

\author{
Anindita Sengupta Ghatak, Gaurav Rawal and Animangsu Ghatak* \\ Department of Chemical Engineering, Indian Institute of Technology Kanpur, Kanpur, UP 208016, India; \\ asengh@iitk.ac.in (A.S.G.); drgaurav@iitk.ac.in (G.R.) \\ * Correspondence: aghatak@iitk.ac.in
}

Academic Editor: Hugo Christenson

Received: 20 July 2017; Accepted: 1 August 2017; Published: 5 August 2017

\begin{abstract}
Nucleation of protein crystals has been shown to be facilitated by substrates decorated with both nano- to micro-scale hierarchical undulations and spatially varying surface potential. In fact, on such surfaces, several proteins were found to crystallize without having to use any precipitant in contrast to all other homogeneous and heterogeneous systems in which precipitant is an essential ingredient for nucleation. While these surfaces were so patterned whole through the area that was brought in contact with the protein solution, it was not clear exactly to what extent the surfaces were required to be patterned to trigger nucleation without use of any precipitant. Here we show that a simple incision may be enough on an otherwise smooth surface for this purpose. In particular, the substrate used here is a smooth silicone film with its surface plasma oxidized to create a thin crust of silica. An incision is then generated on this surface using a sharp razor blade. The silica crust being brittle leads to random nano-microscopic undulations at the vicinity of the incision. These undulations along with surface charge can induce protein crystal nucleation without precipitant.
\end{abstract}

Keywords: protein crystallization; nucleation; nano-wrinkles; incision; surface charges

\section{Introduction}

Crystallization of protein molecules from solution, in general, requires a precipitant to be added. The precipitant is essentially a salt, which in solution shields the charges on the protein, thereby suppressing their electro-static repulsion [1]. Suppression of the repulsion allows protein molecules to come closer and to self-organize into nuclei which eventually grow to become protein crystals. While the charge shielding effect is essential for crystal nucleation, in most cases it is not known a priori the specific salt and its particular concentration in the protein solution at which it would be effective for nucleation. For homogeneous nucleation of a yet-to-be crystallized protein, the extent of super-saturation at which it overcomes the free energy barrier to form the crystal nucleus is not known. When the protein is crystallized on a heterogeneous surface consisting of surface patterns and defects, the free energy barrier for nucleation diminishes, thereby increasing the rate of nucleation [2]. Surface defects include nano-pores, nano-spheres, and particles-like silica particles, polystyrene particles, and so on - which have been found to induce nucleation [3-10]. In fact, nano-pores having a pore diameter similar to that of the radius of gyration of a specific protein [4-8] is found more suitable as nucleant for that specific protein than other surfaces consisting of pores of different other dimensions. Interestingly, for both nano-pores and nano-particles, poly dispersity in sizes is considered important. Surface structures, having a narrow size range, are useful for crystallizing proteins whose radius of gyration closely matches that range. These features however are not very useful for nucleating a yet-to-be crystallized protein, the radius of gyration of which has not yet been determined. In contrast, features having a large range in sizes have the advantage that they can induce nucleation of protein molecules of different sizes. Such substrates are designated as universal 
nucleant [11-15], the large standard deviation in feature size of which has been shown to diminish the energy barrier for nucleation [11]. Not only the size of surface features, but also their shape has been found to influence crystal nucleation [16-18]. Nanopores imprinted on polymeric surfaces have been shown to impose angle specific heterogeneity which leads to specific polymorphs of a compound [16]. Nevertheless, all these features inducing heterogeneous nucleation require a specific precipitant to be used in a specific concentration in solution. In other words, although these substrates facilitate crystal nucleation, uncertainties continue to exist in regard to nucleation of a new protein molecule for which the specific precipitant needs to be found.

It has been shown recently that when heterogeneity in surface topography is coupled with that in surface charges, nucleation occurs without any need of precipitant to be added to the protein solution [19]. The coupled effect of topography and surface charge leads to exceptionally large surface potential gradient that overcomes the effect of charges on the surface of protein molecules so that the substrate itself acts as a precipitant for nucleation. This mechanism of protein crystallization was found to be effective for several protein molecules of wide ranging sizes, thus demonstrating the generality of the effect. Furthermore, most proteins could be crystallized at concentrations far below what is used in conventional methods involving homogeneous nucleation, e.g., hanging drop method. In essence, these surfaces were elastomeric films decorated with self-organized nano-wrinkles with spatially varying surface charges; the charges were generated via plasma oxidation of the surface. While these substrates were patterned all throughout the area which was in contact with the protein solution. In this report, we show that even a single incision drawn on a plasma oxidized surface can induce crystal nucleation without any precipitant.

\section{Experimental}

\subsection{Materials and Method}

Lysozyme (Molecular Weight: $14.4 \mathrm{kDa}$; isoelectric point, pI: 11.35), a protein extracted from chicken egg white and proteinase K (Molecular Weight: $28.9 \mathrm{kDa}$; pI: 8.9) extracted from fungus tritrachium album were purchased from Sigma-Aldrich. Both the proteins were of premium quality (lysozyme: batch no. Sigma L6876 and proteinase K: batch no. Sigma P23308, St. Louis, MO, USA), therefore they were used in experiments as received without any further purification. Trisodium citrate buffer $(\mathrm{pH}=4.8)$ and Tris buffer $(\mathrm{pH}=7.5)$ were procured from Sigma Chemicals and were used for crystallizing lysozyme and proteinase K, respectively. Polydimethylsiloxane (PDMS) (Sylgard 184, procured from Dow Corning, Midland, MI, USA) was used for making the heterogeneous substrates. Microscope glass slides procured from Fisher Scientific (New Hampshire, NH, USA) were used as rigid support on which the substrate was attached. A polystyrene Petri dish procured from Tarson (Kolkata, India) was used for housing the substrate. Thin acrylic sheet of $\sim 6 \mathrm{~mm}$ thickness was used for making the reservoir for buffer. Silane molecule: Octadecyltrichlorosilane (OTS) purchased from Sigma-Aldrich (St. Louis, MO, USA) was used for coating the microscope glass slide with self-assembled monolayer (SAM). A micropipette of capacity 1-10 $\mu \mathrm{L}$ was used for dispensing the protein solution on the substrate. Deionized water obtained from a Milli-Q system (Mumbai, India) was used for all purposes in the experiment.

\subsection{Preparation of Substrate}

Thin films of PDMS were prepared by the method described in [13]. Briefly, a desired quantity of Sylgard 184 elastomer mixed thoroughly with the crosslinking agent in 10:1 w/w ratio was poured between two parallel microscope glass slides coated with a SAM of OTS. The slides were kept separated by two spacers of thickness $350 \mu \mathrm{m}$ and were cured by heating at $80^{\circ} \mathrm{C}$ for two hours. After curing, the glass slides were gently removed to obtain a free, solid PDMS film. Three sets of substrates were prepared by using these films. To prepare set I substrates, the PDMS film was used without any surface modification except that a gentle incision was made on it using a sharp razor blade on; the incision 
was nearly $70 \pm 5 \mu \mathrm{m}$ deep. These substrates were christened $\mathrm{S}_{0 \mid 0}$ and was used as the control. The set II substrates were prepared by oxidizing the PDMS surface with oxygen plasma to form a thin crust of silica and an incision was then made on it as before. These substrates are represented by $\mathrm{S}_{0 \mid 4}$. Here " 4 " in the subscript denotes that the film was oxidized in low power radiofrequency oxygen plasma (Harrick Plasma, model PDC-32G, pressure $\sim 0.05$ Torr, power $\sim 6.8 \mathrm{~W}$ ) for $4 \mathrm{~min}$. The set III samples were prepared, by generating nano-microscopic wrinkles on plasma oxidized PDMS films as depicted in [13]. In brief, a PDMS film was first stretched uniaxially by $\sim 40 \%$ and was then surface plasma oxidized using an oxygen plasma for $\sim 4 \mathrm{~min}$; followed by it, the film was released instantaneously, thereby generating surface wrinkles which had amplitudes of $\sim 30 \mathrm{~nm}$ and wavelength $\sim 300 \mathrm{~nm}$. The wrinkles were most aligned perpendicularly to the direction of the stretching of the film. Also, on this film an incision was made by gently driving a razor blade normal to the direction of stretching of the film, so that the incision was aligned with the nano-wrinkles. These substrates were named as $\mathrm{S}_{40 \% 14}$. These substrates all were gently blown with dry nitrogen gas in order to remove any loosely held debris on the film surface. These films were then imaged using atomic force microscopy (AFM) and were also used for crystallization experiments. It is also worth emphasizing that several samples of each kind of substrate were prepared to check the reproducibility of the results.

\subsection{AFM Imaging of Surfaces}

Bruker multimode 8 AFM (Billerica, MA, USA) was used for scanning the surface of all samples in PFQNM mode using SCANASYST-AIR tip. The tip was first calibrated using sapphire sample to get the deflection sensitivity; the spring constant of the tip was calculated using thermal tuning method.

\subsection{Preparation of Protein Solution}

The desired amount of lysozyme was dissolved in $20 \mathrm{mM}$ trisodium citrate buffer $(\mathrm{pH}=4.8$ ) to prepare a solution containing $10 \mathrm{mg} / \mathrm{mL}$ of protein. The desired amount of proteinase $\mathrm{K}$ was dissolved in $25 \mathrm{mM}$ tris buffer $(\mathrm{pH}=7.5)$ to obtain $10 \mathrm{mg} / \mathrm{mL}$ concentration of protein in the solution. No salt was added as precipitant in either of these two protein solutions.

\subsection{Crystallization Experiment}

In Figure 1, we present a representative image of the crystallization set up; it is described in detail in [19]. In brief, the experiment involves the use of the heterogeneous substrate described earlier. The substrate was physically adhered to a rigid microscope glass slide. A drop of the respective protein solution of volume $\sim 4 \mu \mathrm{L}$ was gently dispensed on the substrate and a second microscope glass slide coated with a SAM of OTS was brought in close proximity to it; two spacers of desired thickness were inserted between the top and bottom glass plates so that a desired gap of $40 \mu \mathrm{m}$ could be maintained between them. In this way, the liquid drop was formed into a sandwiched disk; the curved meniscus of the disk was open to the surroundings and it is through this area that evaporation of water could occur. Because of wettability of the substrate, the meniscus was concave, which is known to cause a smaller extent of evaporation than a convex meniscus or one without any curvature because of kelvin effect. Within each such set of glass plates, one to five liquid disks were formed by dispensing that many number of liquid drops on the substrate. The whole set-up consisting of the pair of glass plates and the liquid disks sandwiched between them were housed inside a polystyrene Petri dish. Inside this Petri dish, we also housed two small pieces of acrylic sheet of suitable thickness with a shallow well grooved into each of them; a pool of the buffer was maintained inside these wells. The Petri dish was sealed with paraffin film so as to maintain humid condition inside it; thus the extent of evaporation of the solvent from the liquid disks could be further controlled by this process. The sealed Petri dish was then kept inside an incubator maintained at $20^{\circ} \mathrm{C}$ temperature. Several such experimental set-ups were prepared for both lysozyme and proteinase $\mathrm{K}$ to examine the variability in observation. In almost all cases, the initial diameter of the liquid disk was $\sim 1.128 \mathrm{~mm}$ and during nucleation of the crystal, it shrank to $\sim 1 \mathrm{~mm}$. 


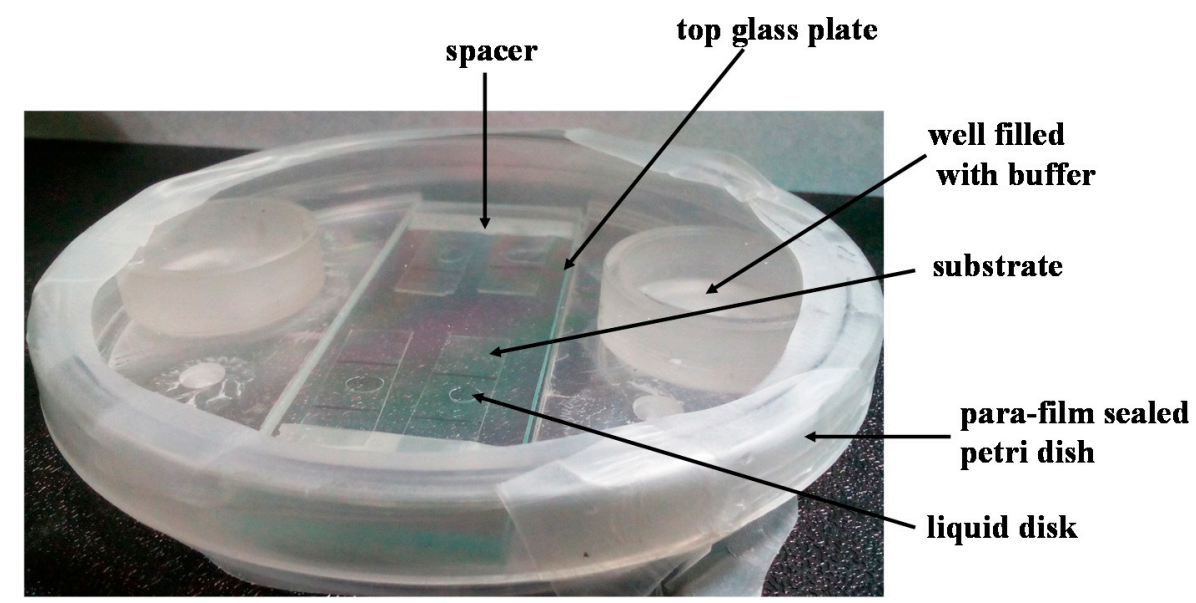

Figure 1. Schematic of crystallization set up.

\section{Results and Discussion}

In Figure 2, we show the AFM image of the surface at the vicinity of the incision for all the three sets of substrates. The 2D and 3D images in Figure 1 shows that for set I samples, the incision was sharp with the film surface remaining smooth at the vicinity of it. However, for set II and III samples, the surface of PDMS film at the vicinity of the incision was far from being smooth, it consisted of nanoto microscopic bumps rendering the surface of the film highly heterogeneous. It is clear that the brittle silica layer underwent deformation and consequent fracture. In fact, the AFM images in Figure $2 \mathrm{~g}-\mathrm{k}$ show that the effect of the incision was confined within lateral distance of $90 \mu \mathrm{m}$ from the incision. A type III substrate was used for capturing these images; the nano-wrinkles can be seen in Figure 2j,k. We will show that the heterogeneities thus generated can be used for crystal nucleation from protein solution in buffer without use of any precipitant. It is also worth noting that although we have focused here on topography of surfaces, it has been described earlier [19] in detail by carrying out Kelvin probe force microscopy (KPFM) of similar such surfaces that these surfaces also consist of negative charges. The surface charges in combination with undulated topography having large curvature leads to large surface potential and gradient in surface potential. Very small surface potential is however generated when the surface remains charged but without any topographical features. 

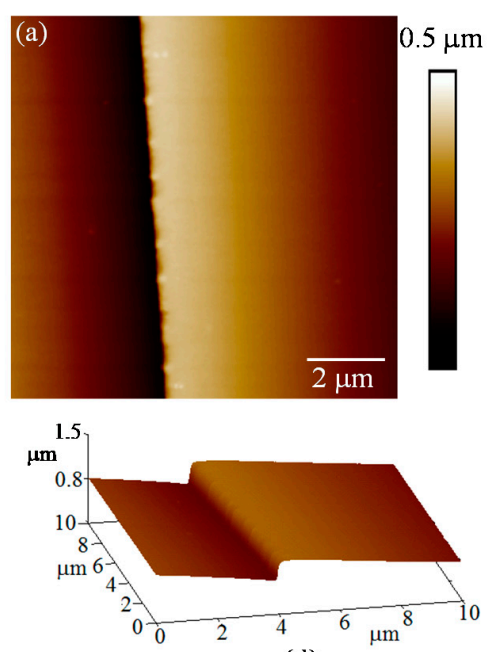

(d)
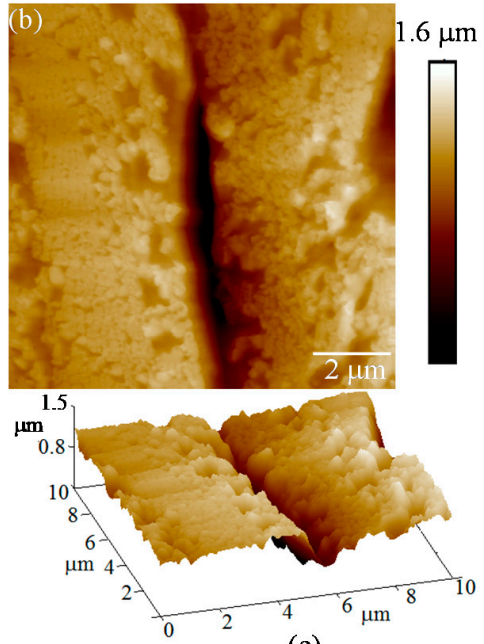

(e)
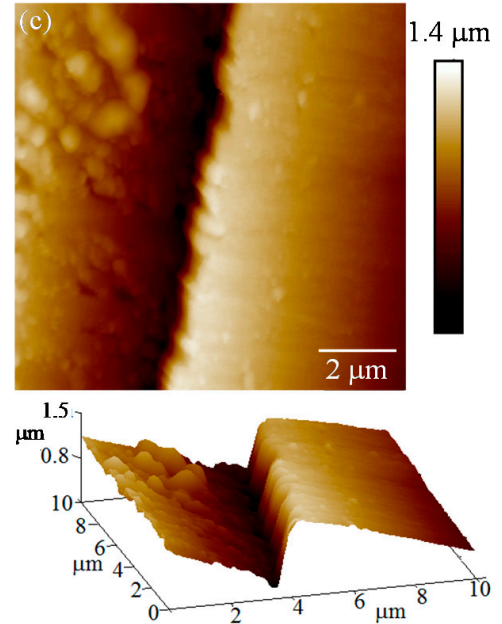

(f)

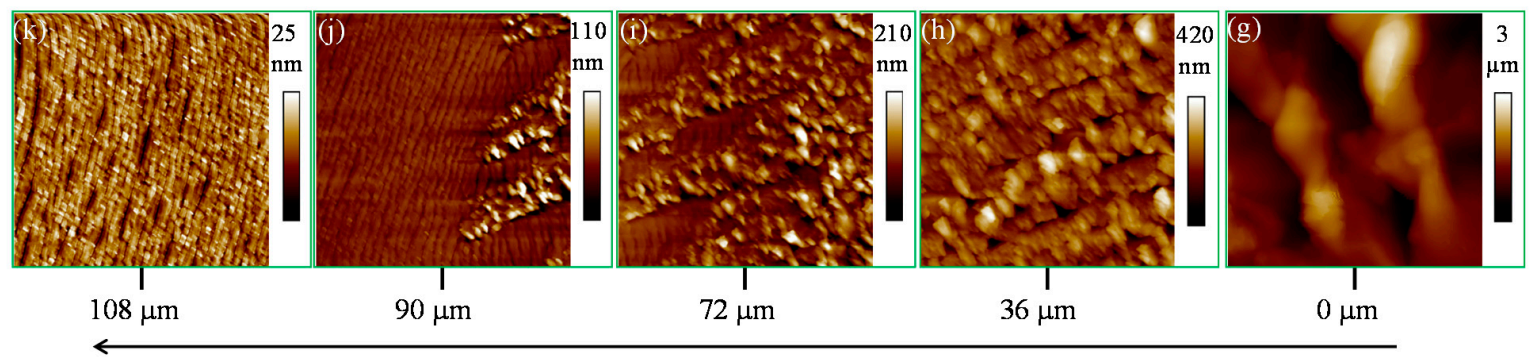

Figure 2. The atomic force microscopy (AFM) images show the profile of PDMS films at the vicinity of an incision. Images $(\mathbf{a}-\mathbf{c})$ correspond to a PDMS film without any surface modification (set I type of substrate), one plasma oxidized for $4 \mathrm{~min}$ (set II type) and one (set III type) consisting of nano- to microscopic wrinkles prepared by the method described in [12]. (d-f) Images represent 3D plots of surfaces corresponding to those in images a-c, respectively. $(\mathbf{g}-\mathbf{k})$ Series of AFM images of surfaces are presented as function of distance from the incision, the location of which is shown in image (g). The bar chart of respective images shows that the roughness of the surface decreases with distance away from incision (as indicated by the arrow).

In Figure 3, we show optical micrographs of crystals of two different protein molecules lysozyme and proteinase $K$ on set $I$ and II substrates. Both the $S_{0 \mid 0}$ and $S_{0 \mid 4}$ substrates were used with and without incision. Figure 3a,e show that no crystal nucleated either for lysozyme or for proteinase $\mathrm{K}$ on the $\mathrm{S}_{0 \mid 0}$ substrate even after 15 days. In a similar way, image $3 \mathrm{~b}$ and $\mathrm{f}$ also show that on $\mathrm{S}_{0 \mid 4}$ substrate also devoid of any incision, crystals did not appear. It is worth noting that $\mathrm{S}_{0 \mid 0}$ surface was devoid of any surface charge, whereas $\mathrm{S}_{0 \mid 4}$ substrate was negatively charged uniformly everywhere because of plasma oxidation [19]. Yet, no nucleation suggests that spatial variation of charges was required for precipitant-free nucleation to occur. This hypothesis was verified in Figure $3 c, d, g, h$ in which on $\mathrm{S}_{0 \mid 4}$ surfaces with the incision few large crystals appeared within $120 \mathrm{~h}$. The crystals were plate shaped in which the third dimension was not visible. It has been shown earlier that the coupled effect of surface pattern and charges lead to large potential gradient that induces protein molecules to self-assemble to form crystals [19-21]. The same mechanism here is expected to be effective because of the disordered topography of the charged surface; in fact, here the potential gradient is expected to be randomly distributed over the area of the substrate which is expected to diminish the energy barrier for nucleation [11,22], thereby facilitating crystallization of proteins of large range of molecular weight. In the present context, both lysozyme with molar mass of $14.3 \mathrm{kDa}$ and proteinase $\mathrm{K}$ with $28.9 \mathrm{kDa}$ could be crystallized using the same surface. Figure $3 \mathrm{c}$ and $\mathrm{g}$ also show that crystals appeared over a large area and not necessarily over the incision or in the vicinity of it. Such observations were made in several sets of experiments (>10) for both lysozyme and proteinase $\mathrm{K}$. The crystals appeared 
also away from the edge of the liquid disk, i.e., from where water could evaporate. This observation implies that crystallization occurred not because of an increase in the concentration of protein unlike in many other examples [23], but because of the effect of the substrate. In fact in Figure 3c, the crystal first appeared away from the edge, the edge of the shrinking disk of liquid later caught up with it. In fact, the appearance of few large crystal suggests that nucleation occurred at a small extent of supersaturation. This aspect was further highlighted in the next set of experiments in which the protein concentration in solution was halved with no addition of precipitant.

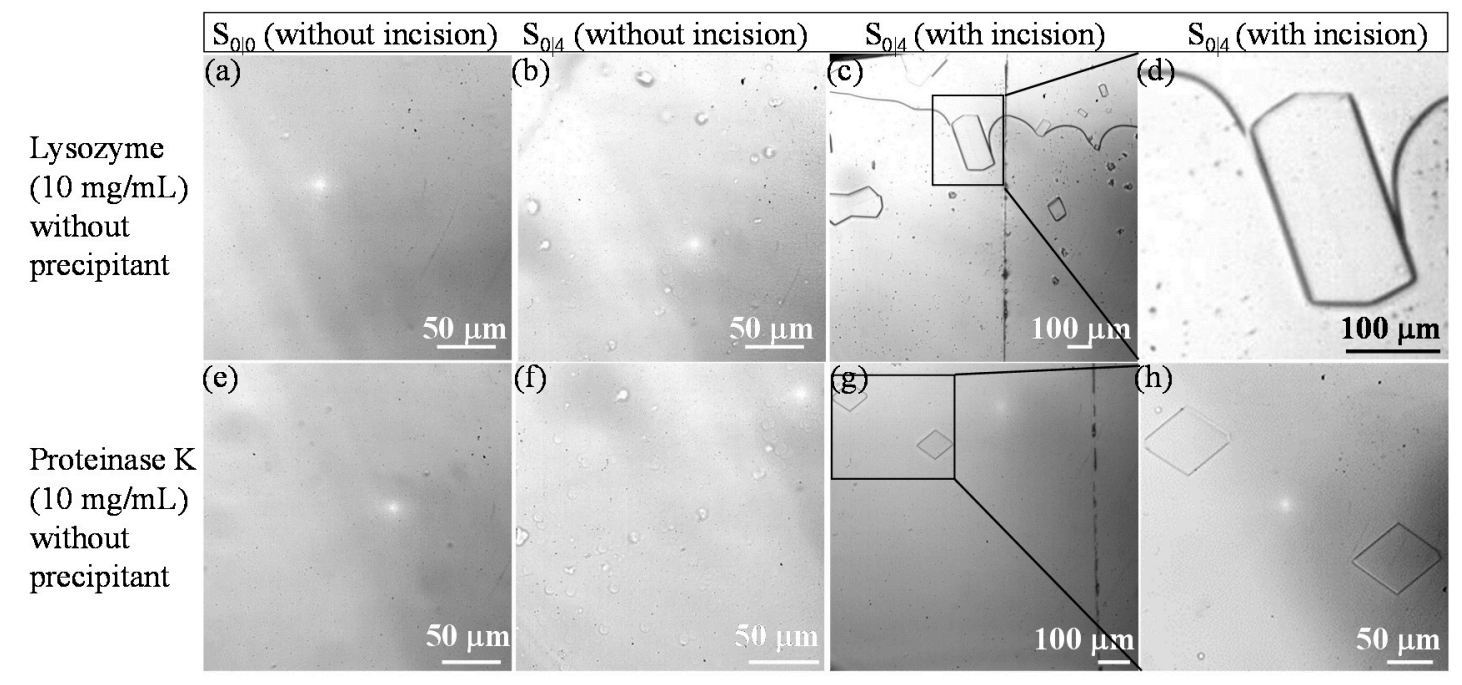

Figure 3. The optical micrographs summarize occurrence of crystals of lysozyme and proteinase K on set I and set II substrates. The concentration of both proteins in respective buffer was $10 \mathrm{mg} / \mathrm{mL}$. Crystals did not appear on set I (images $\mathbf{a}$ and $\mathbf{e}$ ) and set II (images $\mathbf{b}$ and $\mathbf{f}$ ) substrates devoid of any incision. However, crystals of both lysozyme and proteinase K appeared on set II substrates (images c and g) which were decorated with incision. Small portions in images (c) and (g) are magnified in (d) and (h), respectively to show that these crystals were plate shaped without any visible third dimension.

In Figure 4, we show that even at a very small concentration of protein in the solution, the crystals can be nucleated without using any precipitant. Here, both lysozyme and proteinase $\mathrm{K}$ were used at $5 \mathrm{mg} / \mathrm{mL}$ concentration, which to our knowledge is the minimum of what all have been reported in literature [24-26]; apparently, such a low concentration of the protein was smaller than the level of supersaturation. These solutions were used with set III substrates, which were patterned with nano-wrinkles and were decorated with and without any incision. Whereas on $S_{40 \% / 4}$ surfaces without any incision, nucleation was limited to just one or two crystals which grew to assume large size, on the $\mathrm{S}_{40 \% / 4}$ surface with incision, several crystals nucleated. Interestingly, nucleation happened here in the vicinity of the incision in contrast to earlier observation (Figure 3), in which such localization did not happen simply on plasma oxidized surfaces: $\mathrm{S}_{0 \mid 4}$. Thus, the combined effect of nano-wrinkles and incision led to localized occurrence of crystals. Here crystals also appeared far away from that edge, suggesting that not evaporation but surface induced aggregation of protein molecules led to nucleation. It is also possible to control the extent of nucleation and their spatial location where crystals appear by drawing a suitable incision on the substrate. 


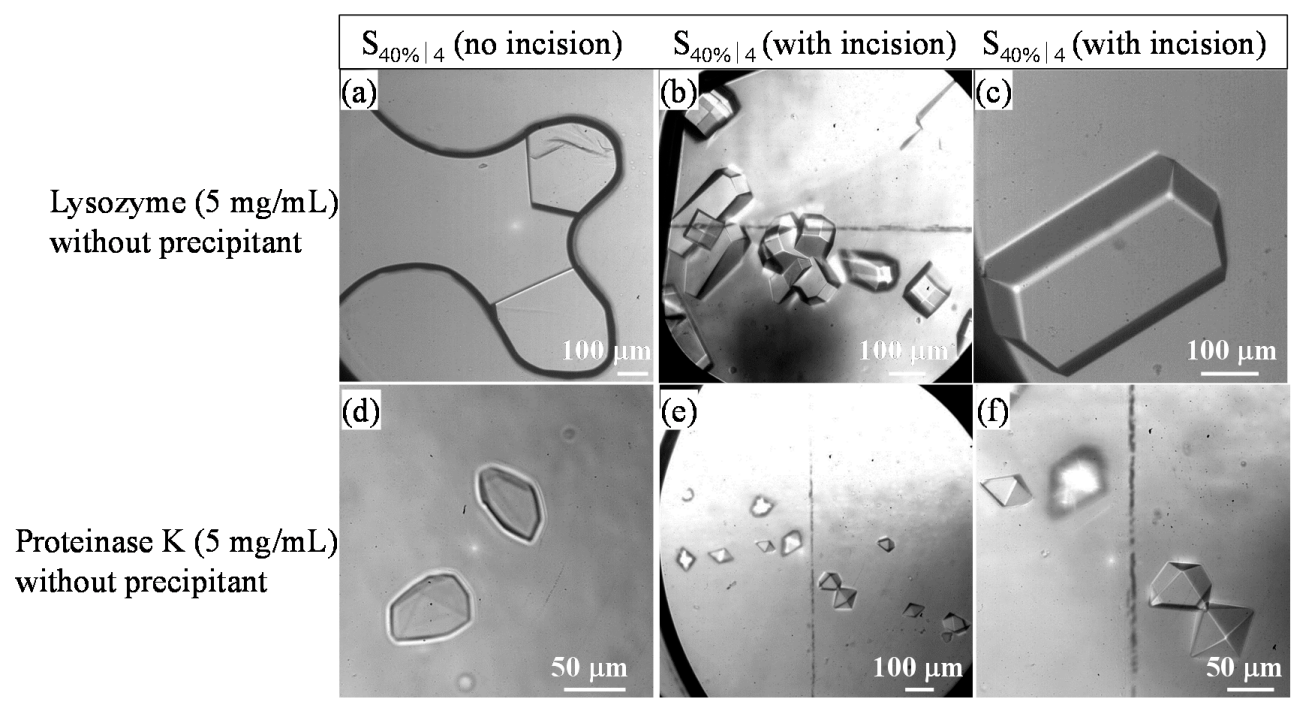

Figure 4. Optical micrographs summarize occurrence of lysozyme $(\mathbf{a}-\mathbf{c})$ and proteinase $\mathrm{K}(\mathbf{d}-\mathbf{f})$ crystals on set III substrates. The concentration of both proteins in respective buffers was $5 \mathrm{mg} / \mathrm{ml}$ and no precipitant was used in either case. Images (a) and (d) depict the appearance of a few crystals of respective proteins on set III substrates devoid of any incision. Images (b) and (e) show appearance of large number of crystals of these proteins on set III substrates decorated with the incision. Selected portions of image (b) and (e) are magnified in images (c) and (f) to clearly show the three dimensional morphology of the crystals.

In order to examine the reproducibility of these results, the crystallization experiments were repeated by generating at least 25 different liquid disks on $12-15$ different $S_{40 \% \mid 4}$ substrate samples. These tests were performed for both lysozyme and proteinase $\mathrm{K}$, both of which crystallized without any precipitant in all the liquid disks. In Figure 5, we present eight different optical images of crystals of each of these proteins from these experiments. These results from repeated experiments demonstrate precipitant-free crystallization of protein molecules on the nano-patterned substrates presented here.

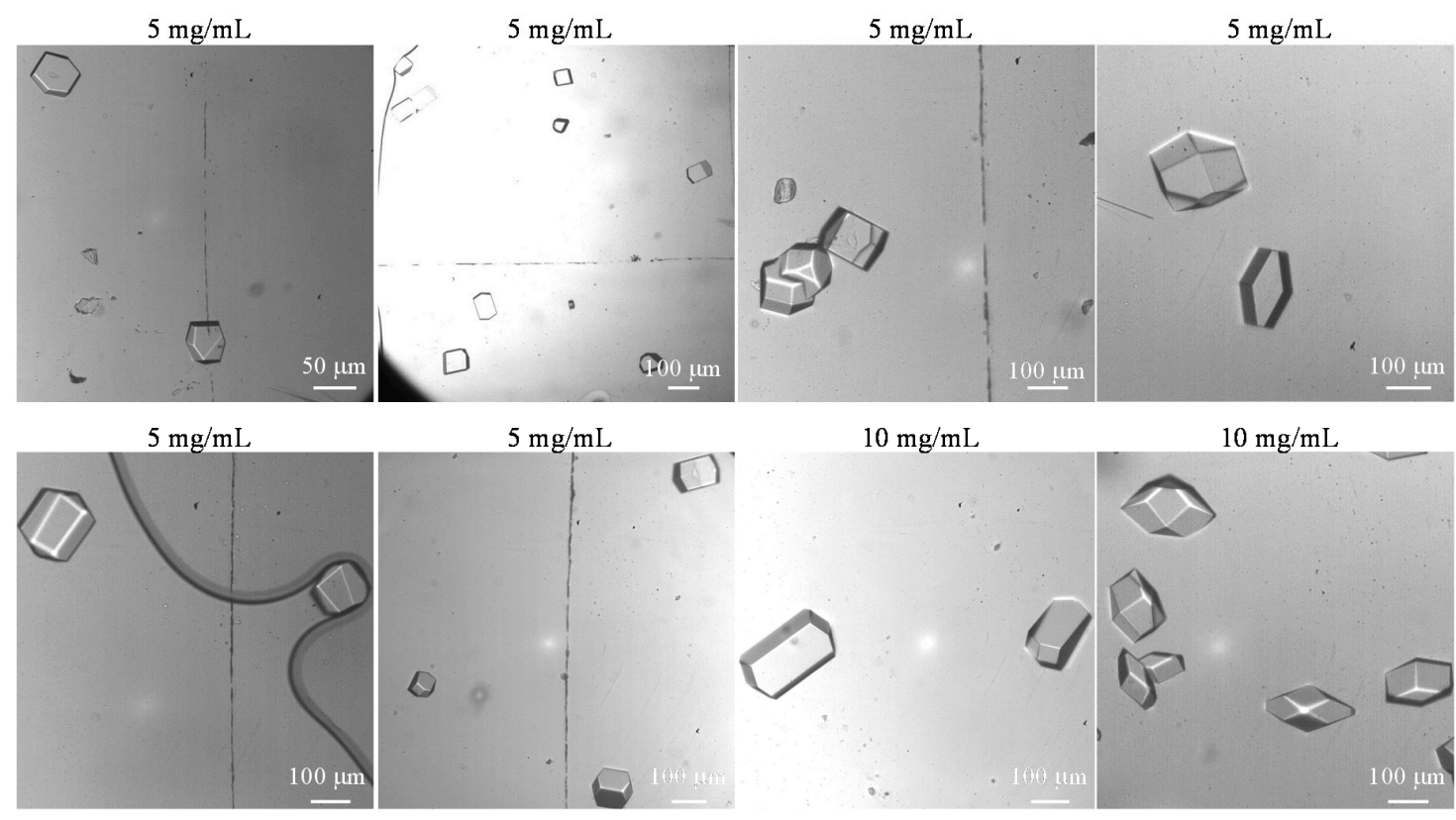

Figure 5. Cont. 


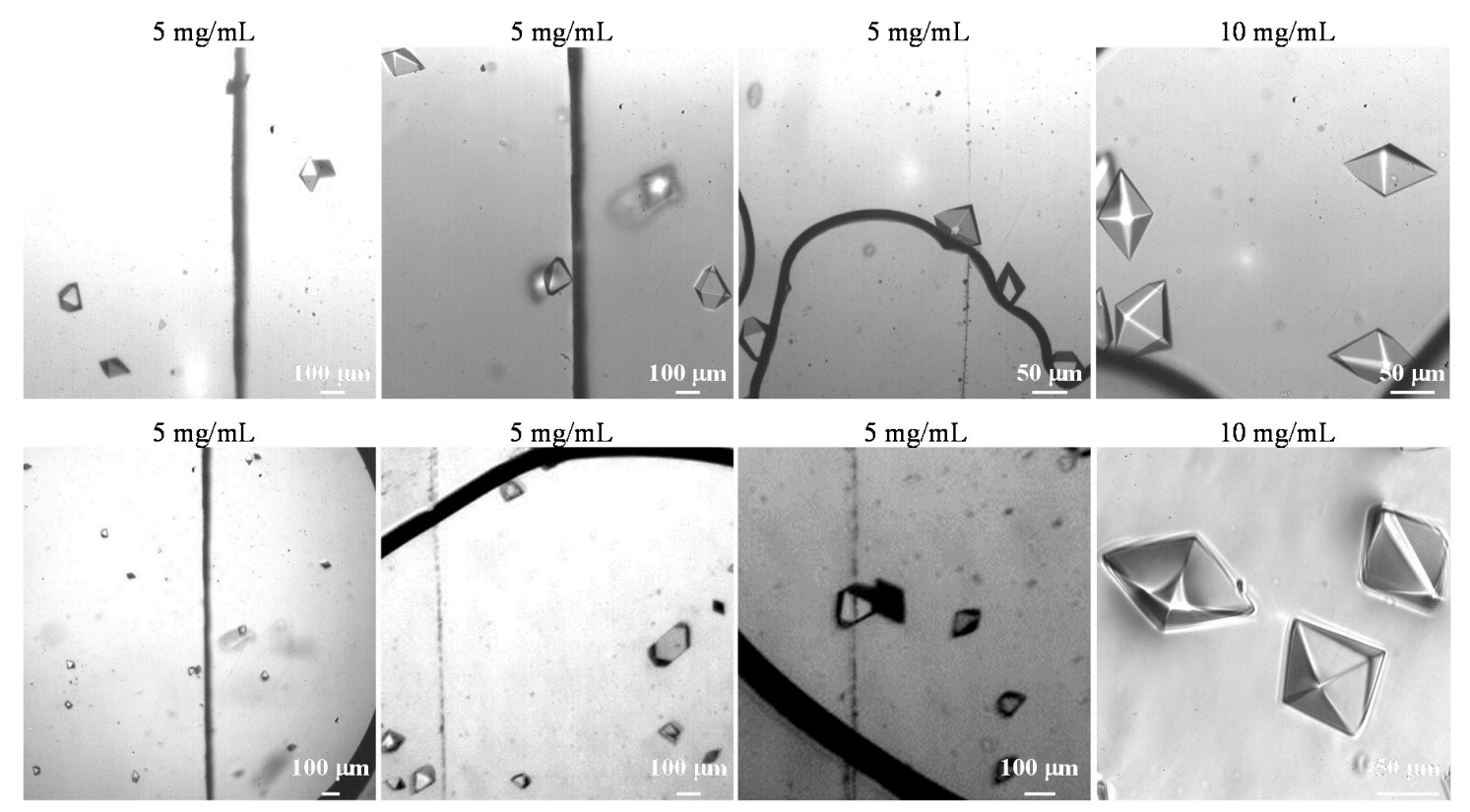

Figure 5. Optical micrographs of lysozyme and proteinase $\mathrm{K}$ from repeated experiments on different samples of the substrate $\mathrm{S}_{40 \% / 4}$ with incision.

\section{Summary}

To summarize, in this report we have explored the effect of incision on plasma oxidized substrate on nucleation of crystals from protein solution. We have shown that when precipitant is not used, no crystal appears if the surface is merely decorated with incisions, as in such cases the surface remains devoid of any charge or topographical heterogeneity. However crystals nucleate, even without precipitant when the surface gets decorated with both surface charges and topographical undulations. These topographical features are created when an incision is made on a plasma oxidized PDMS substrate comprised of a thin and rigid crust. However, such a surface induces very little nucleation if the incision is not made on it, as in such cases, the surface remains charged but without any topographical heterogeneity. In order to explore the coupled effect of nano-heterogeneity of substrate and the incision on crystal nucleation, we first generated nano-wrinkles on the PDMS surface and then made the incision on it. While such a surface induces nucleation, even without the incision, the results in Figure $4 \mathrm{~b}-\mathrm{e}$ show that the coupled effect of incision on nano-wrinkled surfaces leads to the formation of a larger number of crystal nuclei as compared with the surfaces devoid of any incision. Importantly, crystals could be nucleated even for very low concentration of protein in solution. Crystals can also be localized in the vicinity of the incision, thereby opening up the possibility of large scale crystal patterning of organic macromolecules. However, it is worth noting also that it is not clear at this stage if the heterogeneous substrates presented here will be useful for crystallizing other proteins, particularly the yet-to-be crystallized ones. Since commercially purchased proteins have some possibility of containing impurities, it will also be worth working with completely purified proteins to see if they crystallize without any precipitant on these substrates as well.

Acknowledgments: Anindita Sengupta Ghatak acknowledges Department of Science and Technology, Government of India (SB/FTP/ETA-437/2012) and Animangsu Ghatak acknowledges IIT Kanpur (CARE Grant) for financial assistance.

Author Contributions: Anindita Sengupta Ghatak prepared the substrates, carried out protein crystallization experiments, and participated in the writing of the paper; Gaurav Rawal carried out AFM studies on the surfaces; Animangsu Ghatak conceptualized the idea of the experiment and wrote the paper.

Conflicts of Interest: The authors declare no conflict of interest. 


\section{References}

1. McPherson, A. Crystallization of Biological Macromolecules; Cold Spring Harbor Lab. Press: Woodbury, NY, USA, 1999.

2. Debenedetti, P.G. Metastable Liquids; Princeton University Press: Princeton, NJ, USA, 1996.

3. Garcia-Ruiz, J.M. Nucleation of protein crystals. J. Struct. Biol. 2003, 142, 22-31. [CrossRef]

4. Shah, U.V.; Williams, D.R.; Heng, J.Y.Y. Selective crystallization of proteins using engineered nanonucleants. Cryst. Growth Des. 2012, 12, 1362-1369. [CrossRef]

5. Shah, U.V.; Allenby, M.C.; Williams, D.R.; Heng, J.Y.Y. Crystallization of proteins at ultralow supersaturations using novel three-dimensional nanotemplates. Cryst. Growth Des. 2012, 12, 1772-1777. [CrossRef]

6. Holbrough, J.L.; Campbell, J.M.; Meldrum, F.C.; Christenson, H.K. Topographical Control of Crystal Nucleation. Cryst. Growth Des. 2012, 12, 750-755. [CrossRef]

7. Salazar-Kuri, U.; Estevez, J.O.; Antunez, E.E.; Martinez-Aguila, B.S.; Warren, J.B.; Andi, B.; Cerniglia, M.L.; Stojanoff, V.; Agarwal, V. Nucleation of sub-micrometer protein crystals in square-shaped macroporous silicon structures. Cryst. Growth Des. 2015, 15, 2801-2808. [CrossRef]

8. Khurshid, S.; Saridakis, E.; Govada, L.; Chayen, N.E. Porous nucleating agents for protein crystallization. Nat. Protoc. 2014, 9, 1621-1633. [CrossRef] [PubMed]

9. Kallio, J.M.; Hakulinen, N.; Kallio, J.P.; Niemi, M.H.; Kärkkäinen, S.; Rouvinen, J. The contribution of polystyrene nanospheres towards the crystallization of proteins. PLoS ONE 2009, 4, e4198. [CrossRef] [PubMed]

10. Weichsel, U.; Segets, D.; Janeke, S.; Peukert, W. Enhanced nucleation of lysozyme using inorganic silica seed particles of different Sizes. Cryst. Growth. Des. 2015, 15, 3582-3593. [CrossRef]

11. Chayen, N.E.; Saridakis, E.; Sear, R.P. Experiment and theory for heterogeneous nucleation of protein crystals in a porous medium. Proc. Natl Acad. Sci. USA 2006, 103, 597-601. [CrossRef] [PubMed]

12. Ghatak, A.S.; Ghatak, A. Controlled crystallization of macro-molecules using patterned substrates in a sandwiched plate geometry. Ind. Eng. Chem. Res. 2011, 50, 12984-12989. [CrossRef]

13. Ghatak, A.S.; Ghatak, A. Disordered nano-wrinkle substrates for inducing crystallization over a wide range of concentration of protein and precipitant. Langmuir 2013, 29, 4373-4380. [CrossRef] [PubMed]

14. Ghatak, A.S.; Koch, M.; Guth, C.; Weiss, I.M. Peptide induced crystallization of calcium carbonate on wrinkle patterned substrate: implications for chitin formation in molluscs. Int. J. Mol. Sci. 2013, 14, 11842-11860. [CrossRef] [PubMed]

15. Bommineni, P.K.; Punnathanam, S. Enhancement of nucleation of protein crystals on nano-wrinkled surfaces. Faraday Discuss. 2016, 186, 187-197. [CrossRef] [PubMed]

16. Diao, Y.; Harada, T.; Myerson, A.S.; Hatton, T.A.; Trout, B.L. The role of nanopore shape in surface-induced crystallization. Nat. Mater. 2011, 10, 867-871. [CrossRef] [PubMed]

17. Caridi, A.; Kulkarni, S.A.; Di Profio, G.; Curcio, E.; Ter Horst, J.H. Template-Induced Nucleation of Isonicotinamide Polymorphs. Cryst. Growth Des. 2014, 14, 1135-1141. [CrossRef]

18. Lounaci, M.; Chen, Y.; Rigolet, P. Channel height dependent protein nucleation and crystal grow thin microfluidic devices. Microelectron. Eng. 2010, 87, 750-752. [CrossRef]

19. Sengupta Ghatak, A.; Ghatak, A. Precipitant-free crystallization of protein molecules induced by high surface potential. J. Cryst. Growth Des. 2016, 16, 5323-5329. [CrossRef]

20. Al-Haq, M.I.; Lebrasseur, E.; Choi, W.K.; Tsuchiya, H.; Torii, T.; Yamazaki, H.; Shinohara, E. An apparatus for electric-field-induced protein crystallization. J. Appl. Cryst. 2007, 40, 199-201. [CrossRef]

21. Koizumi, H.; Fujiwara, K.; Uda, S. Control of nucleation rate for tetragonal hen-egg white Lysozyme crystals by application of an electric field with variable frequencies. Cryst. Growth Des. 2009, 9, 2420-2424. [CrossRef]

22. Tosi, G.; Fermani, S.; Falini, G.; Gavira, J.A.; Garcia Ruiz, J.M. Hetero- vs Homogeneous Nucleation of Protein Crystals Discriminated by Supersaturation. Cryst. Growth Des. 2011, 11, 1542-1548. [CrossRef]

23. Ikezoe, Y.; Kumashiro, Y.; Tamada, K.; Matsui, T.; Yamashita, I.; Shiba, K.; Hara, M. Growth of Giant Two-Dimensional Crystal of Protein Molecules from a Three-Phase Contact Line. Langmuir 2008, 24, 12836-12841. [CrossRef] [PubMed]

24. Curcio, E.; Fontananova, E.; Di Profio, G.; Drioli, E. Influence of the structural properties of poly(vinylidene fluoride) membranes on the heterogeneous nucleation rate of protein crystals. J. Phys. Chem. B 2006, 110, 12438-12445. [CrossRef] [PubMed] 
25. Ebeling, W.; Hennrich, N.; Klockow, M.; Metz, H.; Orth, H.D.; Lang, H. Proteinase K from Tritirachium album Limber. Eur. J. Biochem. 1974, 47, 91-97. [CrossRef] [PubMed]

26. Annunziata, O.; Payne, A.; Wang, Y. Solubility of lysozyme in the presence of aqueous chloride salts: common-ion effect and its role on solubility and crystal thermodynamics. J. Am. Chem. Soc. 2008, 130, 13347-13352. [CrossRef] [PubMed] 